\author{
Cristian PĂUNA, PhD Candidate \\ E-mail: cristian.pauna@ie.ase.ro \\ Professor Ion LUNGU, PhD \\ E-mail: ion.lungu@ie.ase.ro \\ Economic Informatics Doctoral School \\ The Bucharest University of Economic Studies
}

\title{
PRICE CYCLICALITY MODEL FOR FINANCIAL MARKETS. RELIABLE LIMIT CONDITIONS FOR ALGORITHMIC TRADING
}

\begin{abstract}
Trading the financial markets is a common idea nowadays. Millions of market participants, individuals, companies or public funds are buying and selling different equities in order to obtain profit from the buy and sell price difference. Once the equity was established, the main question marks are when to buy, when to sell and how long to keep the opened positions. This paper will present a mathematical model for the cyclicality of the price evolution. The model can be applied for any equity in any financial market, using any timeframe. The method will gives us information about when is good to buy and when is better to sell. The price cyclicality model is also a method to establish when the price is approaching to change its behavior in order to build limit conditions to stay away the market and to minimize the risk. The fundamental news is already included in the price behavior. Being exclusively a mathematical model based on the price evolution, this method can be easily implemented in algorithmic trading. The paper will also reveal how the cyclicality model can be applied in automated trading systems and will present comparative results obtained in real-time trading environment.
\end{abstract}

Keywords: financial markets (FM), price cyclicality (PCY), algorithmic trading (AT), high frequency trading (HFT), automated trading software (ATS).

\section{JEL Classification: M15, O16, G23}

\section{Introduction}

"A financial market is a market in which people trade financial securities, commodities, and value at low transaction costs and at prices that reflect supply and demand" (Wikipedia, 2018). The choice of equities to be traded is a particular issue. The market participants have their own strategies to chose what to trade depending on their investment objectives, economical status of the equities, economical events, fundamental and financial news etc. Once the idea to trade a specified financial market seems to be a good one, the next step is to analyze the

DOI: $10.24818 / 18423264 / 52.4 .18 .10$ 
Cristian Păuna, Ion Lungu

price level. Based on the price history and volatility, a considerable number of trading strategies have been invented in order to determine when is the right moment to buy or to sell equity. In AT "the defined sets of rules are based on timing, price, quantity or any mathematical model." (Seth, 2014) This paper will present one of these strategies, based on a mathematical model to reflect the price cyclicality for any financial market.

"The markets are complex adaptive systems exhibiting unpredictable behavior." (Leshik and Cralle, 2011). Even so, the price cyclicality model starts from the hypothesis that the price behavior has a cyclical evolution, both in the long run and in the shorter time intervals. This model is based on the idea that the price can be associated with a wave, with variable wavelengths. The wave theory is not something new. It was successfully applied to develop many mathematical models in engineering. The idea seems to be right thinking how the market participants act when the price of the equity is increasing: more investors start to buy that equity and after a while, some of them start to sell in order to close the profitable positions. In that time interval the price evolution slows down. At a certain moment of time more participants start to close their positions, more than those who buy then and a reversed tendency will be present sooner or later in the price behavior. This is a cyclical phenomenon and it is our basic idea. The unpredictable behavior will be reflected by the variation of the wavelengths and the amplitude of the price movements.

The method proposes to find a mathematical function to describe this behavior based only on the price evolution and to reveal those time intervals when the price is approaching to change its behavior. This model will be practically a mathematical transformation function of the price into a wave function in order to establish the cyclical behavior of the price movements. Once the transformation was applied to the price, this model will reveal the right moments of time when is good to buy or to sell the financial instrument. More important, using the asymptotical evolution of the model, the method will permit to set some limit conditions in order to determine the time intervals when is better not to trade and to stay away the market.

To make a complete image for the cyclicality model, this paper will also present how the functional parameters of this indicator can be optimized, how the cyclicality model can be applied in different time frames and how much is the correlation coefficient in order to trust the method. On the last part, this paper will present some real trading results obtained with and without the limit conditions built with the cyclicality model in order to reveal the power of this methodology.

\section{Price cyclicality model}

The model construction starts considering a time price series and two moving averages, one with $p_{1}$ period and the second with $p_{2}$ period, where $p_{1}<p_{2}$. For each moment of time $(i)$, the values of the moving averages will be 
Price Cyclicality Model for Financial Markets. Reliable Limit Conditions for Algorithmic Trading

noted $M_{a i}$ and $m_{a i}$, where $M_{a i}$ is the moving average with the lower period and $m_{a i}$ is the moving average with the higher period. On the normal uptrend of price: $M_{a i}>m_{a i}$. When the trend is reversed and the price goes down, the positions of the two moving averages are changing and we will have: $M_{a i}<m_{a i}$. The calculation methodology for moving averages is not a subject of this paper and can be found in any mathematical encyclopaedia starting with (Cox, 1961). For the price cyclicality model any type of moving average can be used, differences will be revealed later in this paper.

It is known that in the normal up trend of a price evolution, a moving average is going also in the up direction and when the trend price is reversed, the moving average is decreasing. Being a function with different signs of the first derivate on the end of an interval, there must to be a point with a root of the first derivate on that interval, considering that the function is a derivable function on the entire interval. With this property, the moving average will present a maximum point in this interval, shifting from a positive derivate to a negative one.

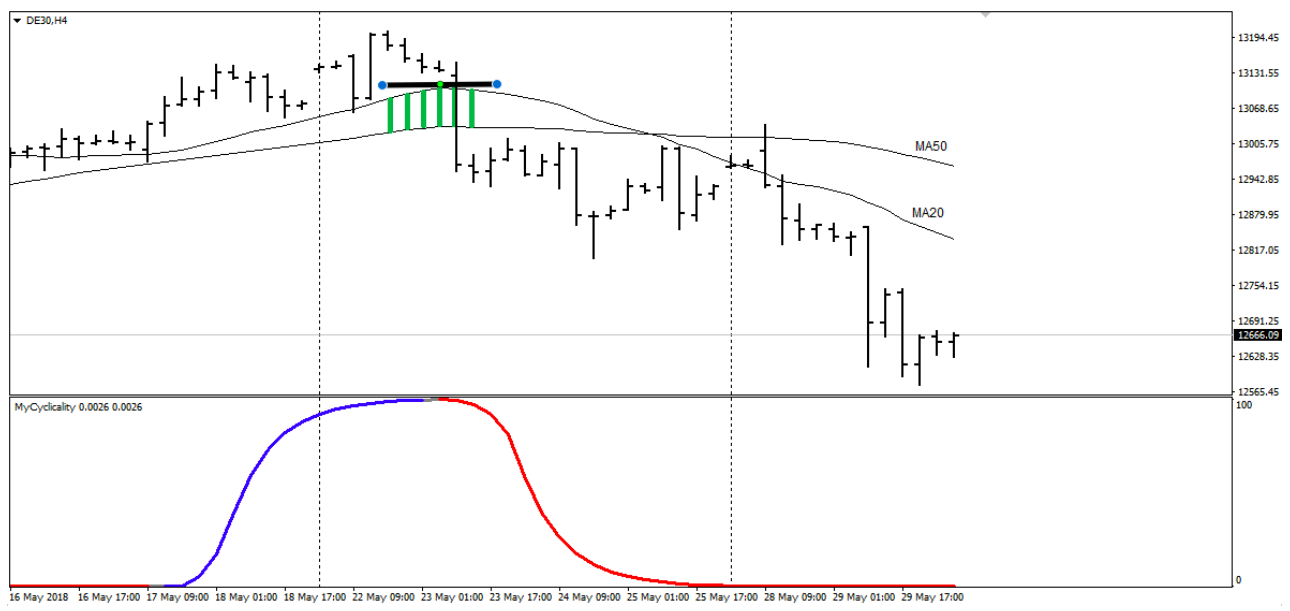

Figure 1. Moving average behavior as basic for the price cyclicality model.

Looking at figure 1, another aspect can be observed: the distance between the two moving averages is increasing until a moment of time. After the maximum of the Mai, this distance will decrease and the moving averages will be intersected after a time period. All of these geometrical observations give us the possibility to consider that a trend change in the price behavior will be accompanied by a similar change in the evolution of the distance between two moving averages. The results of the model will prove the hypotheses. 
Looking at figure 1, it can be observed that in the time interval before the trend change, usual the price is higher than Mai. This means for some known trading strategies that the trend is still up. On that interval the distance between the two moving averages is decreasing. For our model this means a price behavior change is approaching. If we will consider a function as the ratio of the distance between the two moving average and the difference between the maximum and the minimum distance the two moving averages recorded on an interval we will have a function which describe the evolution of that distance in time. Let's consider the maximum and minimum of the distance between the two moving averages on an $n$ time interval:

$$
\min _{i}=\min _{k=i}^{i-n}\left(M a_{k}-m a_{k}\right) \text { and } \max _{i}=\max _{k=i}^{i-n}\left(M a_{k}-m a_{k}\right)
$$

where $i$ represent the time step and $n$ is called the period for our model, meaning the subset of intervals from the price history taken into account by our method. Reporting the current distance between the two moving averages to the difference between $\min _{i}$ and $\max _{i}$ we will obtain a limited function:

$$
\Delta_{i}=\frac{\max _{i}-\xi_{i}}{\max _{i}-\min _{i}} \text { where } \xi_{i}=M a_{i}-m a_{i}
$$

There are many mathematical models to describe a wave phenomenon. Different degrees polynomial functions can be used for this case. Working with small intervals and computing the results for each one gives us the possibility to build the cyclicality as first order Spline function (Berbente, Mitran si Zancu, 1997) given by:

$$
P C Y_{i}=\alpha\left(\Delta_{i}-P C Y_{i-1}\right)+P C Y_{i-1} \text { where } P C Y_{0}=0
$$

The function used in formula (3) is a first order polynomial function. On a short time interval this will be a line between two consecutive points. Including the distance between the two moving averages in formula (2) of our model, the function (3) will have a cyclical form on a longer interval just because of the price behavior. The function graph is revealed in the figure 1 . We can see that when the price behavior makes the moving averages to move away one of each other, the cyclicality function gives us a positive evolution. After the maximum distance between the two moving averages is passed, the negative price evolution will be accompanied by a negative evolution of the cyclicality function.

The model used generates a limited function of cyclicality between 0 and 100 and this function will present an asymptotic behavior near its maximum and 
Price Cyclicality Model for Financial Markets. Reliable Limit Conditions for Algorithmic Trading

minimum point when the price is near the point where will change the direction. This asymptotical evolution is given by the distance between the two moving averages, reported to the maximum distance on that interval. As we can see in the figure 1, in the increasing intervals of the cyclicality function the price has an up evolution, meaning on those intervals is good to buy the equity because the price is increasing. Similarly, when the cyclicality function turns on red (decreasing), a down movement for the price will come, meaning the long positions can be closed and the equity must to be sold. The correlation coefficient between the price movements and the monotony of the cyclicality function will gives us trust in order to use this relation.

An advantage of this method is that when the cyclicality function tends to take the maximum value in that asymptotical behavior, the price is already in a limited interval and the position can be closed before the price to turn in the descending interval, in order to maximize the profit and to reduce the risk.

\section{Functional parameters}

The functional parameters of the price cyclicality model presented are: the type of moving average, the two periods of moving averages $p_{1}$ and $p_{2}$ and the functional parameter $\alpha$ which will be named the gradient.

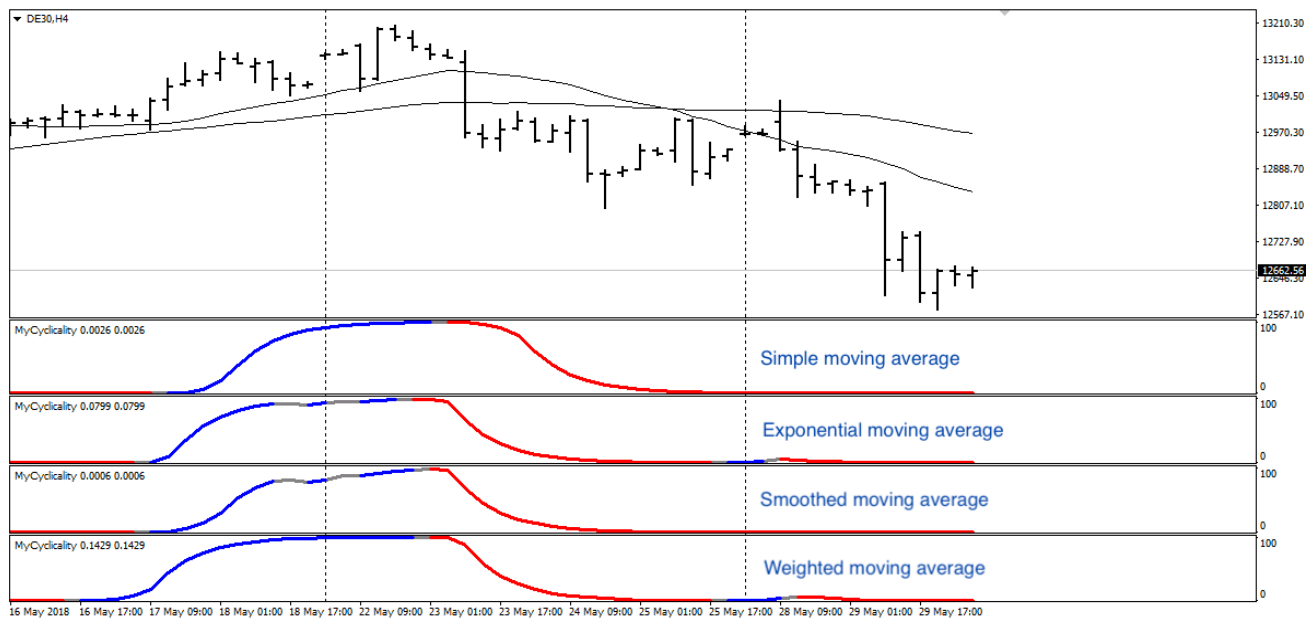

Figure 2. Influence of moving average types in the price cyclicality model

Regarding the type of moving averages, any type can be used in order to build the price cyclicality function. As we can see in figure 2, the less lag is presented by the usage of exponential moving average, but sometimes the model is not too accurate because of some undecided monotony of the obtained function. To avoid this, the simple moving average is usual used, especially when the PCY 
model is combined with other trading strategies only to confirm the price movement and to predict the changing in the market behavior.

The periods of the two moving averages used in the model are important factors in order to optimize the model. These two parameters can be the subject of an optimization and machine-learning process and can be different from a traded equity to other. To reveal the influence of these two factors we present the figure 3:

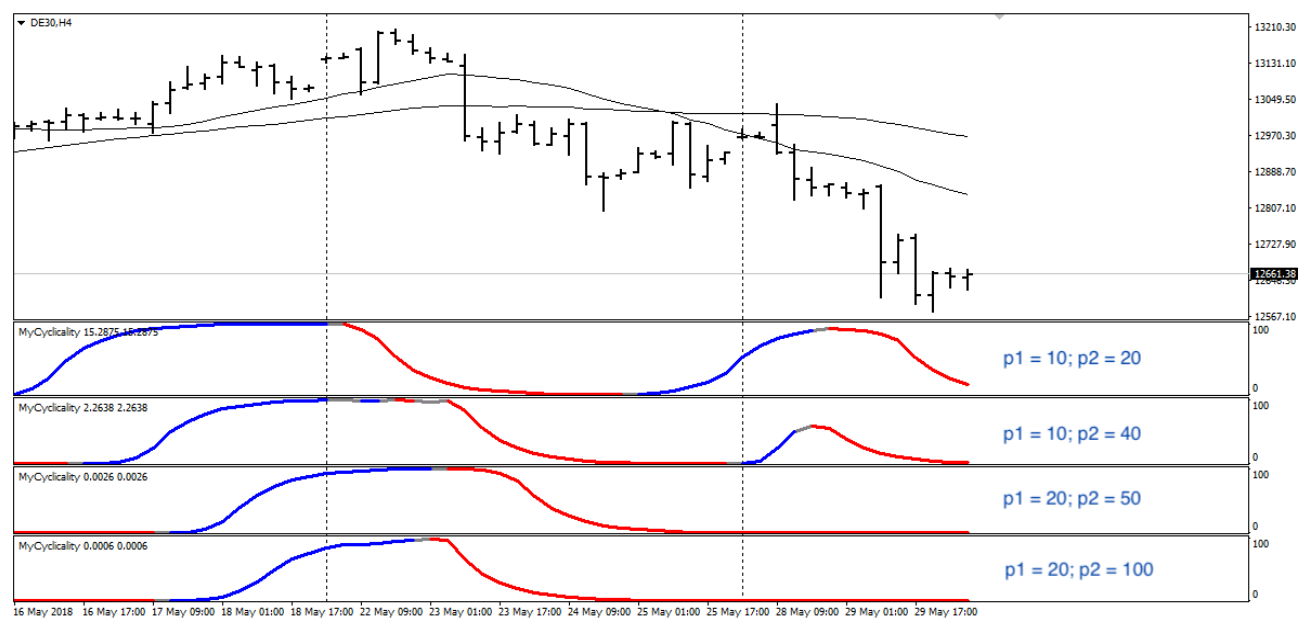

Figure 3. Influence of moving average periods in the price cyclicality model

The parameter $\alpha$ determines the curvature of the price cyclicality function. This parameter is at our choice. In the practice the normal value for the gradient parameter is less or equal with 0.33 . As we can see in the figure 4, for lower values of $\alpha$ we will obtain a smoothed evolution for the PCY function which will permit to have longer trades as and to delay the exit position in order to increase the profit.

The period $\mathrm{n}$ is the number of time intervals used to apply the PCY model. This is also a functional parameter which can be optimized. Usual this period is set between 10 and 20 and all other parameters are optimized depending on it. For lower values of $n$ the precision of the method Is decreasing. For higher values of $n$ the computational power needed is increasing, without a significant improvement of the precision of the model. 
Price Cyclicality Model for Financial Markets. Reliable Limit Conditions for Algorithmic Trading

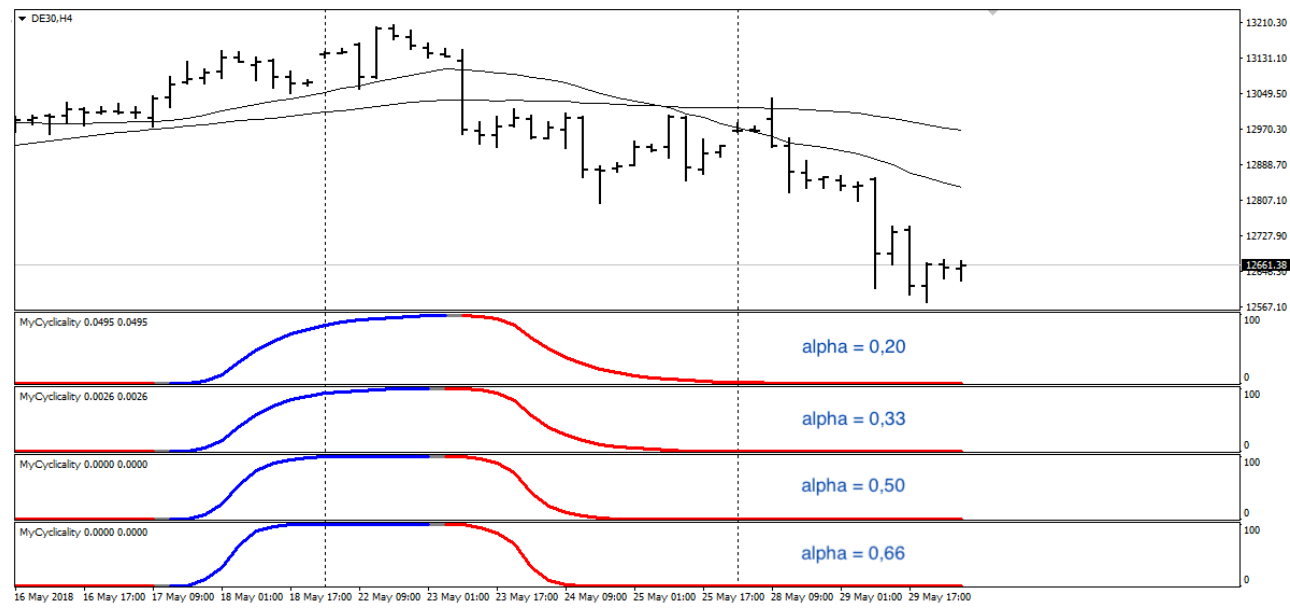

Figure 4. Influence of gradient parameter in the price cyclicality model

\section{Different time frames}

The PCY model includes two moving averages. As we can see in the equations presented, the time frame is not included anywhere except in the two moving averaged used. This means the model can be easily applied to any timeframe, depending on how it is represented the time price series for those $\mathrm{n}$ time intervals. The PCY has no limitation and can be applied on any timeframe. In figure 5, we present the model applied to M5, M15, M30, H1, H4 and D1 timeframes.

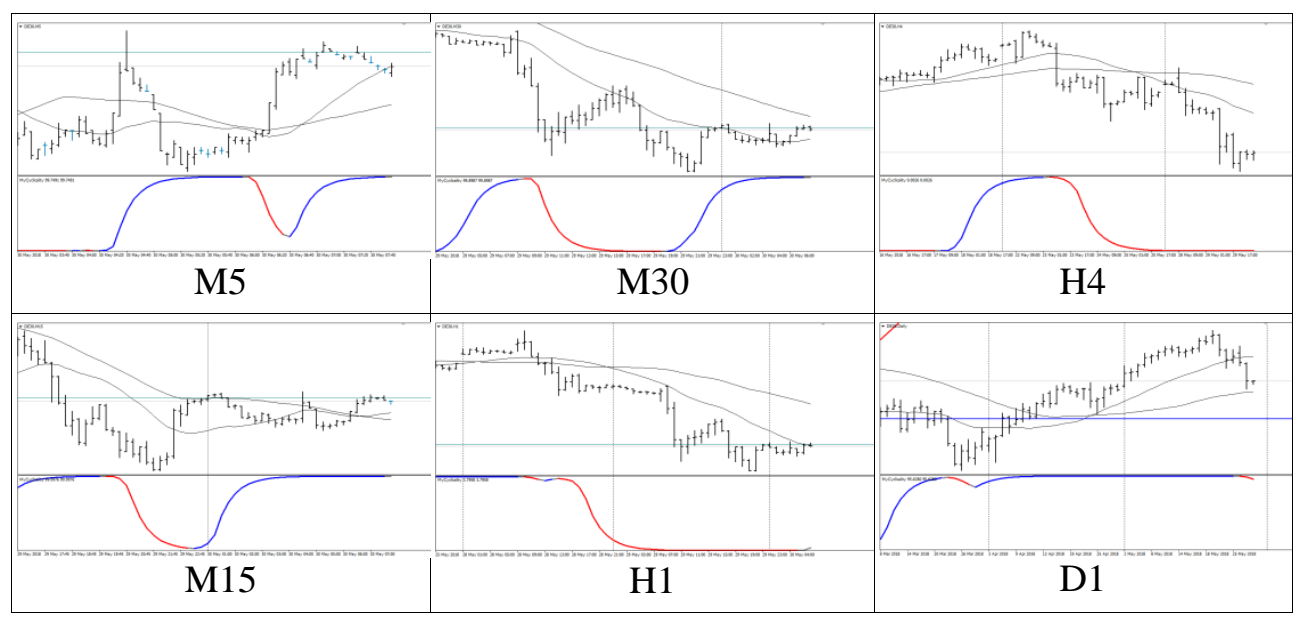

Figure 5. The price cyclicality model in different time frames 


\section{Cristian Păuna, Ion Lungu}

A very simple trading strategy can use only PCY indicator. When the PCY function turns blue in all timeframes presented, a buy signal can be considered. This strategy will gives us a very good entry point in the market.

\section{Correlation coefficient}

As we explained, we use the cyclicality function in order to establish if the price is in an upwards trend or in a descending period. This will gives us a criterion to consider buy or sell trades in the equity market. In order to see how correlated are the price movement and the PCY function we have to analyze the correlation coefficient between the two. Using the Pearson correlation formula:

$$
r=\left(\sum_{i=1}^{N}\left(\left(x_{i}-X\right)\left(y_{i}-Y\right)\right)\right) /\left(\sqrt{\sum_{i=1}^{N}\left(x_{i}-X\right)^{2}} \sqrt{\sum_{i=1}^{N}\left(y_{i}-Y\right)^{2}}\right)
$$

where

$$
\begin{aligned}
& x_{i}=1 \text { if } \text { price }_{i}>\text { price }_{i-1} \text { and } x_{i}=0 \text { if } \text { price }_{i}<\text { price }_{i-1} \\
& y_{i}=1 \text { if } P C Y_{i}>P C Y_{i-1} \text { and } y_{i}=0 \text { if } P C Y_{i}<P C Y_{i-1}
\end{aligned}
$$

and $X$ and $Y$ are the averages for the $x_{i}$ and $y_{i}$ variables on the $N$ historical interval. We can calculate the value of the $r$ coefficient at any time using the historical price values. Because we work with a time price series, computing the value of $r$ on a specified time interval length must be made for the entire interval of price values, in order to establish the maximum and the minimum values of the $r$ coefficient on the entire historical period taken in the study. For a ten years interval, between January 2008 and May 2018 we have obtained the results presented in the table 1 for different financial markets.

Based on the results in the table 1, it can be said that it is a positive strong correlation between the price movements and the direction of the $P C Y$ function. The results in the table 1 are obtained computing the results for the period mentioned above by using all timeframes mentioned at chapter 4 (M5, M15, M30, $H 1, H 4$ and $D 1$ ) for $p_{1}=20, p_{2}=50, \alpha=0.33$ and $n=10$. The correlation coefficient depends on the timeframe but all values remain between the values presented in the table 1 . 
Price Cyclicality Model for Financial Markets. Reliable Limit Conditions for Algorithmic Trading

Table 1. The PCY correlation coefficient with the price in different markets

\begin{tabular}{|c|c|c|}
\hline Stock index & $\mathrm{r}$ - minimal value & $\mathrm{r}$ - maximal value \\
\hline DAX30 [6] & 0.70711 & 0.99612 \\
\hline FTSE100 [7] & 0.70817 & 0.99691 \\
\hline CAC40 [8] & 0.70517 & 0.99317 \\
\hline SMI20 [9] & 0.70166 & 0.99948 \\
\hline DJIA30 [10] & 0.70081 & 0.99879 \\
\hline NASDAQ100 [11] & 0.70712 & 0.99751 \\
\hline S\&P500 [12] & 0.81649 & 0.99901 \\
\hline ASX200 [13] & 0.70152 & 0.99831 \\
\hline NIKKEI225 [14] & 0.84444 & 0.99961 \\
\hline
\end{tabular}

The strong positive correlation between the price movement and the $P C Y$ function gives us a sustained reason to say that the $P C Y$ function is a good price predictor. When the $P C Y$ function has a minimum value and turn into the ascending period, an up price movement is expected. The minimal point of the $P C Y$ function will be a good buy signals. Consequently the maximum point of the $P C Y$ function will be a good signal for a short trade or at least to close the long ones. The price correlation with the $P C Y$ function works well in the majority of cases. However, when the high impact financial news arrives unexpectedly, the correlation coefficient can take lower values, but these values are higher 0.77 meaning we still have a good correlation between the $P C Y$ function and the price movement.

\section{Limit conditions}

As presented, the $P C Y$ function minimal and maximal points are a good indicator for a buy or a sell trade. These trading conditions can be automated and included in an ATS because the model is based only on mathematical calculation of the $P C Y$ function, which basically includes only the time price series values. Once a long trade was opened after a minimal point of the $P C Y$ function, the question is how long that trade can be opened. The intension is to keep the trade open as longer as possible in order to maximize the profit, but the decision must to close the trade on profit, before the price turn. The $P C Y$ function permits to automate this decision. 


\section{Cristian Păuna, Ion Lungu}

Using the $P C Y$ function we can set up some conditions in order to close the profitable trades before the price reversal. We will call these conditions as limit conditions, once the trade will be kept open until that limit value of the $P C Y$ function will be met. As it was shown, the $P C Y$ function has an asymptotic behavior. After a moment of time, the gradient of the $P C Y$ function is decreasing and the function values tend to touch the 100 level. The limit conditions can be set on the $P C Y$ function value with:

$$
\left\{\begin{array}{l}
\text { closebuy trades if } P C Y_{i}>\text { LongLimit } \\
\text { closesell trades if } P C Y_{i}<\text { ShortLimit }
\end{array}\right.
$$

The values set for the LongLimit and ShortLimit are at the algorithmic trader disposal. Usual in the current practice the limits are:

$$
\left\{\begin{array}{c}
99<\text { LongLimit }<99.9 \\
1>\text { ShortLimit }>0.1
\end{array}\right.
$$

The limit conditions on $P C Y$ values are very easily to be implemented in any AT or HFT procedure. The used values for each limit can be subject of an optimization process for any trading strategy, depending on the financial market traded, depending on the time frame used and depending on the risk level.

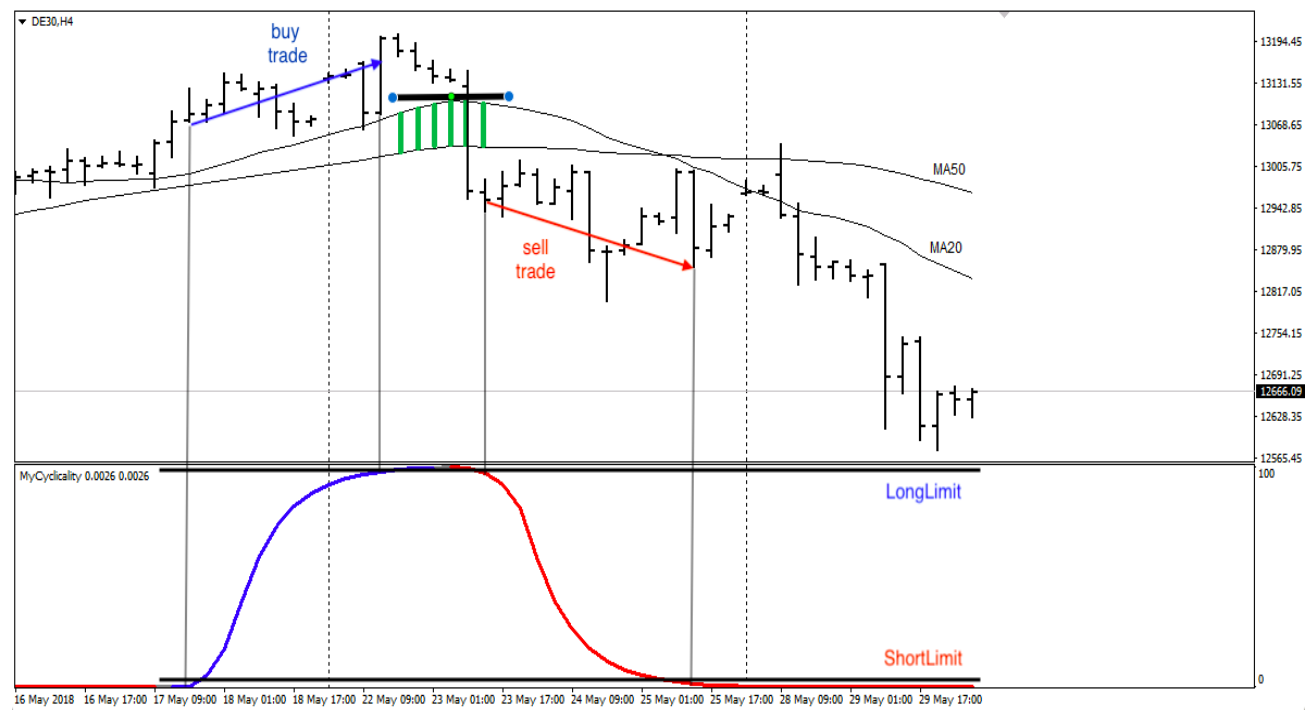

Figure 6. Limit conditions to trade with the price cyclicality model 
Price Cyclicality Model for Financial Markets. Reliable Limit Conditions for Algorithmic Trading

In figure 6, there are presented a buy and a sell trades based on $P C Y$ function. The buy trade was opened after the minimum value of the $P C Y$ and closed with the LongLimit condition. The sell trade was opened by after the maximum point of the $P C Y$ and closed by the ShortLimit. As we can see in figure 6 , the price continued to fall after the close of the sell trade. The limit conditions give us a good mark in order to close the trade before a price turn. Depending on the market behavior, the functional parameters of the $P C Y$ function can be optimized in order to maximize the profit and to minimize the risk involved.

Another kind of limit conditions that can be made using the $P C Y$ function is a filter that can be combined with any other buy or sell signal in order to reduce the risk. For any trading signal depending on the price: $\operatorname{Signal}\left(p_{i}\right)$, the additional conditions imposed by the $P C Y$ will be given by:

$$
\left\{\begin{array}{l}
\text { buy Signal }\left(p_{i}\right) \text { only if } P C Y_{i}>P C Y_{i-1} \\
\text { sell Signal }\left(p_{i}\right) \text { only if } P C Y_{i}>P C Y_{i-1}
\end{array}\right.
$$

In this way, the monotony of $P C Y$ function will be used as data-mining filter in order to execute only that trades which correspond with the up or down movement of the $P C Y$ function. This filter is a very reliable one and can be used regardless of the trading signal.

\section{Trading results with limit conditions}

The $P C Y$ function and the limit conditions built with this model can be combined with any other trading strategy in order to obtain a lower drawdown and to optimize the risk to reward ratio $(R R R)$. In this section we will present some trading results obtained with and without the limit conditions with the $P C Y$ function. In order to reveal the filtering efficiency obtained by using the limit conditions presented in formula (6) and (8), we add some trading results made with the DaxTrader (Păuna, 2010), an ATS which uses the $P C Y$ methodology in order to select the trades. In table 2 , there are presented the trading results of a strategy with and without the PCY filter imposed with formula (8). The results are illustrated in figure 7.

Table 2. Trading results obtained with and without PCY filter

\begin{tabular}{|c|c|c|c|c|c|c|}
\hline & $\begin{array}{c}\text { Total } \\
\text { trades }\end{array}$ & $\begin{array}{c}\text { Profit } \\
\text { /Loss }\end{array}$ & Drawdown & RRR & $\begin{array}{c}\text { Absolute } \\
\text { drawdown }\end{array}$ & $\begin{array}{c}\text { Absolute } \\
\text { RRR }\end{array}$ \\
\hline Without (8) & 427 & 10314 & 14082 & $1: 0.73$ & 11883 & $1: 0.86$ \\
\hline With (8) & 77 & 6597 & 2045 & $1: 3.22$ & 346 & $1: 19.06$ \\
\hline
\end{tabular}



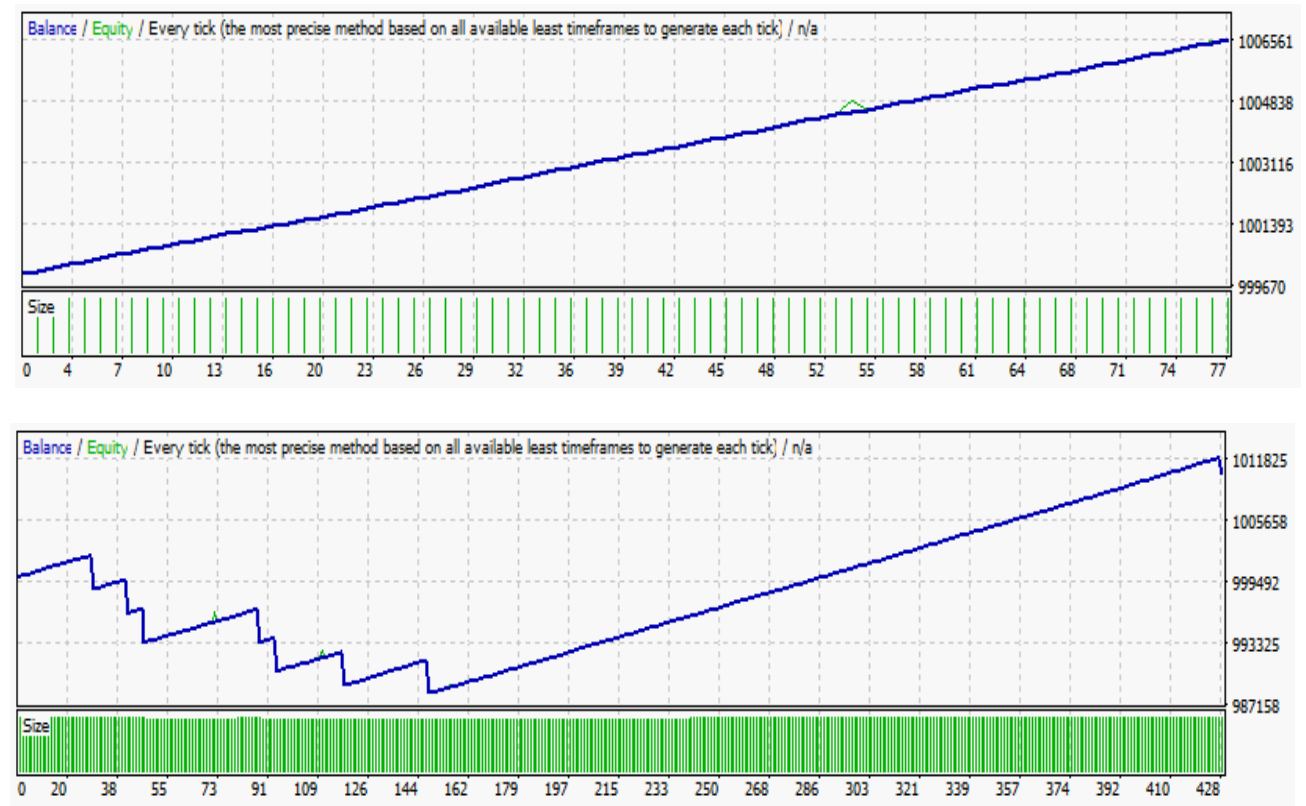

Figure 7. Trading results obtained with and without PCY filter

As we can see, by using the formula (8) for the $P C Y$ filter, the number of trades is considerable lower than the case without the $P C Y$ filter. Even so, the RRR is much lower (1:3.22 instead 1:0.73). Both tests have been used the same trading volume, the same risk and the same trading strategy. The risk management was made using the "Global stop loss method" (Păuna, 2018). Using the filter, the volume can be increased and the profit will be much higher on the same RRR.

In table 3 , there are presented the trading results of a strategy with and without the limit conditions made by formula (6). On this example the LongLimit value used was 99.9 and the ShortLimit value was 0.01 . The results are illustrated in figure 8.

Table. 3. Trading results obtained with and without PCY filter

\begin{tabular}{|c|c|c|c|c|c|c|}
\hline & $\begin{array}{c}\text { Total } \\
\text { trades }\end{array}$ & $\begin{array}{c}\text { Profit } \\
\text { /Loss }\end{array}$ & Drawdown & RRR & $\begin{array}{c}\text { Absolute } \\
\text { drawdown }\end{array}$ & $\begin{array}{c}\text { Absolute } \\
\text { RRR }\end{array}$ \\
\hline Without (6) & 27 & -1161 & 2173 & $-1: 1.87$ & 4627 & $-1: 0.34$ \\
\hline With (6) & 19 & 1581 & 1035 & $1: 1.52$ & 303 & $1: 5.21$ \\
\hline
\end{tabular}


Price Cyclicality Model for Financial Markets. Reliable Limit Conditions for Algorithmic Trading
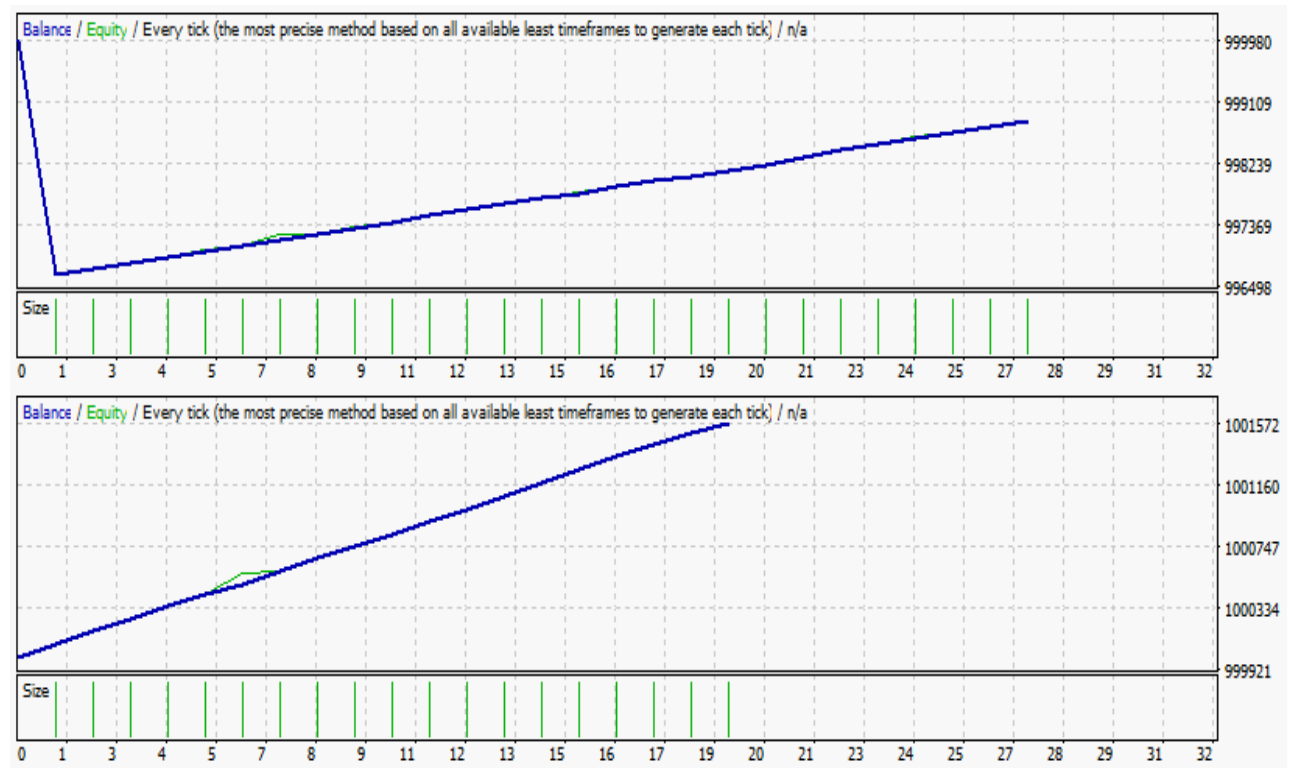

Fig. 8. Trading results obtained with and without PCY filter

\section{Conclusions}

The $P C Y$ is a mathematical model which describes the wave character of the price in a financial market. The model accuracy is confirmed by the high values of the correlation coefficient. This proves a strong and direct correlation between the price movements and the values of the $P C Y$ function. The method can be used in any timeframe for any equity market.

The model is based only on the time price series values and can be easily implemented in any AT or HFT system. The number of the functional parameters of the $P C Y$ method is reduced. This is a significant advantage in order to adapt this method to any ATS. All these functional parameters of the model can be optimized for each market in order to maximize the profit and to minimize the risk.

The $P C Y$ function permits to identify the trend change in the price behavior. Buy and sell trading signals can be automatically assembled in real time after a minimum or a maximum local point of the $P C Y$ function is occurred.

The $P C Y$ model also permits to set limit conditions in order to filter any other trading strategy. The strong correlation between the monotony of the $P C Y$ function and the price local trend gives us a very good trading signal filter proved by the obtained results. The significant improvements of the financial efficiency of trading with the $P C Y$ conditions validate the hypothesis assumed for the model. By simplicity, the cyclicality limit conditions are easily to be used in order to exit the trades made with any type of trading strategy. After the exit wit $P C Y$ limit conditions, new trades in the same direction will not be opened, even the trend is still unchanged, until the $P C Y$ function is not changing its monotony interval, 
Cristian Păuna, Ion Lungu

considering that the asymptotical behavior of the $P C Y$ function is assimilated with the price that is near a maximum or minimum local point.

\section{REFERENCES}

[1] Wikipedia Encyclopedia (2018), Financial Market; Available: https://en. wikipedia.org/wiki/financial market, May 1, 2018;

[2] Seth, S. (2014), Basics of Algorithmic Trading; Investopedia Journal, Available: https://www.investopedia.com/articles/active-trading/101014/basics-algorithmic-tradingconcepts-and-examples.asp, May 1, 2018;

[3] Leshik, E., Cralle, J. (2011), An Introduction to Algorithmic Trading: Basic to Advanced Strategies; Wiley Trading, 2011 - ISBN: 978-1-119-97509-0;

[4] Cox, D.R. Sir (1961), Prediction by Exponentially Weighted Moving Averages and Related Methods; Journal of the Royal Statistical Society; Series B, Vol. 23, No. 2, pp. 414-422;

[5] Berbente, C., Mitran, S., Zancu, S. (1997); Metode numerice, Tehnică Publishing, 1997, ISBN 973-31-1135-X;

[6] Wikipedia Encyclopedia, (2018), Deutsche Aktienindex (DAX30), Available: https://en.wikipedia.org/wiki/DAX;

[7] Wikipedia Encyclopedia, (2018), Financial Times Stock Exchange (FTSE100), Available: https://en.wikipedia.org/wiki/ FTSE_100_Index;

[8] Wikipedia Encyclopedia, (2018), Cotation Assistée en Continu Index (CAC40), Available: https://en.wikipedia.org/wiki/CAC_40;

[9] Wikipedia Encyclopedia, 2018, Swiss Market Index (SMI20), Available: https://en.wikipedia.org/wiki/Swiss_Market_Index;

[10] Wikipedia Encyclopedia, (2018), Dow Jones Industrial Average (DJIA30),

Available: https://en.wikipedia.org/wiki/ DowJonesIndustrialAverage;

[11] Wikipedia Encyclopedia, (2018), National Association of Securities Dealers

Automated Quotations (Nasdaq100), Available: https://en.wikipedia.org/ wiki/NASDAQ100 ;

[12] Wikipedia Encyclopedia, (2018), Standard \& Poor's (S\&P500), Available: https://en.wikipedia.org/wiki/S\%26P_500_Index;

[13] Wikipedia Encyclopedia, (2018), Australian Securities Exchange Index (ASX200), Available: https://en.wikipedia.org/wiki/S\&P/ASX_200;

[14] Wikipedia Encyclopedia, (2018), Nippon Stock Average (Nikkei225), Available: https://en.wikipedia.org/wiki/Nikkei_225;

[15] Păuna, C. (2010), The DaxTrader Automated Trading Software Online

Presentation, Available: https://pauna.biz/thedaxtrader, May 20, 2018;

[16]Păuna, C. (2018), Capital and Risk Management for Automated Trading Systems;

Proceeding of the 17th International Conference of Informatics in Economy, 2018, pp.183189. Available at: https://pauna.biz/ideas;

[17] Mircea Constantin Scheau, Stefan Pop Zaharie(2017), Methods of Laundering

Money Resulted from Cyber-crime. Economic Computation and Economic Cybernetics Studies and Research; Volume 51; No. 3; ASE Publishing, Bucharest;

[18] Dungaciu Dan, Cristea Darie, Dumitrescu Diana Alexandra, Pop Stefan Zaharie (2018), Stratfor vs. Reality (1995-2025). Dilemmas in Global Forecasting; Romanian Journal of Economic Forecasting, Volume 21, Issue 1/ 2018. 